\title{
BMPER is a conserved regulator of hematopoietic and vascular development in zebrafish
}

\author{
Martin Moser ${ }^{1}$, Qingming Yu, Christoph Bode, Jing-Wei Xiong ${ }^{2, \S}$, and Cam Patterson ${ }^{3, \S}$ \\ ${ }^{1}$ University of Freiburg, Internal Medicine III, Germany \\ ${ }^{2}$ The Nephrology Division, Massachusetts General Hospital, Harvard Medical School, Boston, MA \\ ${ }^{3}$ Carolina Cardiovascular Biology Center, University of North Carolina at Chapel Hill, NC
}

\section{Abstract}

For the proper development of vertebrate embryos as well as for survival of the adult organism, it is essential to form a functional vascular system. Molecules involved in this process are members of highly conserved families of proteins that exert conserved functions across species. Bone morphogenetic proteins (BMP) are extracellular factors that are regulated by extracellular modulators and bind to BMP receptors, which in turn activate intracellular signaling cascades. BMPs are necessary not only for induction of endothelial and hematopoietic lineages but also for further endothelial and hematopoietic cell differentiation. Previously, we identified BMPER (BMP endothelial cell precursor derived regulator) and demonstrated its spatiotemporal expression at sites of vasculogenesis and direct modulation of BMP activity. To directly investigate the role of BMPER in vascular development, we cloned the BMPER ortholog in zebrafish (zbmper). It is expressed at sites of high BMP activity, including vascular precursor cells located in the aortic arches and the intermediate cell mass during zebrafish embryonic development. Knockdown of zbmper results in a dorsalized phenotype, a reduced number of gatal expressing hematopoietic precursor cells and of circulating blood cells as well as in a vascular phenotype. The generation of the caudal vein is compromised and the pattern guiding of the intersomitic vessels is disturbed, indicating that zbmper is required for early steps in vascular pattern formation and hematopoiesis in zebrafish.

\section{Keywords}

developmental biology; gene expression; blood vessel formation; hematopoiesis; zebrafish; bone morphogenetic protein; BMPER

\section{Introduction}

For the proper development of vertebrate embryos as well as for survival of the adult organism it is essential to form a functional vascular system. Upon integration of orchestrated signals

\footnotetext{
(c) 2007 Elsevier Ltd. All rights reserved.

Correspondence: Martin Moser, University of Freiburg, Internal Medicine III, Hugstetter Strasse 55, 79106 Freiburg, Germany, E-mail: Martin.Moser@ uniklinik-freiburg.de, phone:++497612793401; fax:++497612703254, Cam Patterson, University of North Carolina at Chapel Hill, Division of Cardiology, CB\# 7075, 130 Mason Farm Road, Bioinformatics Building, 4th Floor, Chapel Hill, NC 27599-7075, E-mail: cpatters@med.unc.edu, Phone: (919) 843-6477; FAX: (919) 843-4164.

\$These authors contributed equally to the work

Publisher's Disclaimer: This is a PDF file of an unedited manuscript that has been accepted for publication. As a service to our customers we are providing this early version of the manuscript. The manuscript will undergo copyediting, typesetting, and review of the resulting proof before it is published in its final citable form. Please note that during the production process errors may be discovered which could affect the content, and all legal disclaimers that apply to the journal pertain.
} 
from early molecular pathways, such as the bone morphogenetic protein (BMP) family (reviewed in $\left[{ }^{1}\right]$ ), spatial and temporal differentiation of mesodermal precursor cells begins, resulting in the formation of the first hemangioblasts $\left[{ }^{2}\right]$. This putative cell population serves as a progenitor for both the hematopoietic and the endothelial lineage. Physical forces as well as a constellation of ligands, receptors, and proteases contribute to the maturation of the vascular tree and subspecification of vascular beds $\left[{ }^{3}\right]$. Given the central role of blood vessel formation in embryogenesis and adult survival, it is not surprising that the involved molecules, such as the BMPs, are highly conserved across species. They are not only involved in the induction of vascular precursor cells, but also play essential roles in other cell fate decisions such as embryonic axis formation. BMPs are extracellular proteins that bind to receptors to exert their functions. Their local activity is influenced by interactions with extracellular modulators such as chordin, a well characterized antagonist for BMP4 [ $\left.{ }^{4}\right]$.

Overexpression of BMPs or BMP receptors results in increased capillary tube formation [ ${ }^{5}$ ]. Under experimental conditions, recombinant BMP2 produces a large increase in size and number of tumor blood vessels, an effect that can be inhibited by specific antagonists [ $\left.{ }^{6}\right]$. In a targeted deletion model for BMP4, most mouse embryos die around ED 7.5 with defects in mesoderm formation and patterning. The few embryos that survive this developmental stage die at ED 9.5 and display a vascular phenotype with reduced numbers of blood islands [ ${ }^{7}$ ].

Furthermore, mutations on the type II receptor BMPR-II in humans have been associated with the development of primary pulmonary hypertension as a result of vascular dysfunction. Similarly, hereditary hemorrhagic teleangiectasia is caused by mutations in the TGF- $\beta /$ BMP pathway (Alk1, endoglin) (reviewed in $\left[{ }^{8}\right]$ ) and can be recapitulated in the respective lack of function mouse models $[9,10]$. These observations as well as loss of function studies of transcription factors dependent on BMP signaling ${ }^{11}$ ] indicate that BMPs exert a stimulating effect on endothelial (precursor) cell activation, migration and proliferation and are necessary not only for induction of mesoderm but also for further vascular differentiation.

Previously, we identified BMPER (BMP endothelial cell precursor derived regulator) in a screen for differentially expressed proteins in endothelial precursor cells $\left[{ }^{12}\right]$. BMPER is a secreted protein that contains 5 cysteine rich domains followed by a von Willebrand D domain and a trypsin inhibitor domain, which is expressed in the AGM region. This region hosts vascular precursor cells during mouse development. BMPER interacts directly with the BMPs 2, 4, and 6 and antagonizes BMP4 activity in Smad reporter gene assays. Using the Xenopus embryo assay, we confirmed antagonistic activity of BMPER towards BMP4 when BMPER RNA was injected ventrally. Additionally, the number of endothelial cells generated in the BMP4 dependent ES-cell differentiation assay can be suppressed by BMPER. Nevertheless, data from studies of Drosophila crossveinless-2 and vertebrate BMPER, which are orthologous proteins, point to a more complicated mechanism of action. With respect to BMP modulation, contradictory activities have been reported. When overexpressed, BMPER inhibits BMP activity by direct interaction with BMPs $\left[{ }^{12-14}\right]$. In loss of function experiments, inactivation of BMPER results in decreased BMP activity, leading to the conclusion that BMPER has proBMP activity under these conditions $\left[{ }^{15,16}\right]$. Cleavage of BMPER protein may be a major determinant of the swith between its pro- versus anti- BMP activity [ $\left.{ }^{16}\right]$.

Collectively, available data indicate that BMPER is expressed at the time and site of vasculogenesis and controls BMP activity by acting as an "internal" BMP modulator of vascular precursor cells, but its specific in vivo role in this context has not been clearly defined. In order to investigate the role of BMPER in vertebrate vascular development, we searched for BMPER orthologs in zebrafish. The zebrafish model allows for easy manipulation of molecular pathways using the morpholino knockdown technique. It is also an excellent model for studies of vascular and hematopoietic formation, providing tools that allow for direct observation of 
the formation of hematopoietic and vascular cells. Here we show that BMPER is an important regulator in blood vessel formation and hematopoiesis.

\section{Materials and Methods}

\section{Morpholino sequences and injections}

Morpholino phosphorodiamidate oligonucleotides (MOs), were produced by Gene Tools (Philomath, OR). The MOs are designed to inhibit translation or splicing of the zbmper RNA (Genbank GB accession number DQ386454). We used two different MOs to knockdown zbmper function with the following sequences: zbmper MO1: TTGGATGATCTTACCGGTGATTAAA; zbmper MO2:

CCAAAGTATCCCAAGCCTGTAGAAA. MO1 targets the first exon-intron splicing site of zbmper mRNA and MO2 is directed against the untranslated region. All MOs were prepared and injected as described $\left[{ }^{17}\right]$. One or two cell stage embryos were injected with the indicated amount of either $\mathrm{MO}$ and raised at $28.5^{\circ} \mathrm{C}$ until analyzed. Zbmper protein was quantified in zebrafish embryo lysates using standard western blotting techniques and an antibody from R\&Dsystems.

\section{Raising and staging of embryos}

Zebrafish were raised and maintained according to standard protocols at $28.5^{\circ} \mathrm{C}\left[{ }^{18}\right]$. Embryos were staged using standard morphologic criteria prior to analysis [ $\left.{ }^{19}\right]$. Blood vessel nomenclature was applied according to published recommendations. $\left[{ }^{20}\right]$. To facilitate visualization of GFP or RNA expression during in situ hybridization $0.003 \%$ phenylthiourea (PTU, Sigma) was added to egg water before $24 \mathrm{hpf}$ to block pigment formation of the embryos. Dorsalization of embryos was classified according to Mullins [ $\left.{ }^{21}\right]$.

Transgenic flk1:GFP and gatal:GFP zebrafish lines were kindly provided by Dr. Shou Lin (University of California, Los Angeles) and used to study hematopoiesis and vascular development. Flk1:GFP fish express GFP driven by the $f l k 1$ promoter in endothelial (precursor) cells and the endocardium allowing for direct observation of the vasculature $\left.{ }^{22}\right]$. Gatal:GFP fish express GFP driven by the gatal promoter in hematopoietic stem cells of the intermediate cell mass $\left[{ }^{23}\right]$.

\section{Cloning, sequening, bioinfomatic analyis, and expression of zbmper}

The full length zbmper cDNA was identified based on mouse BMPER $\left[{ }^{12}\right]$ and cloned by $5^{\prime}$ RACE and 3'RACE PCR (Clontech RACE kit) using day-2 embryo RNA as a template. Gene specific primers were derived from a partial genomic sequence (NA 19100) that was identified by BLAST search of the zebrafish genome against the mouse BMPER sequence. The cDNA sequence obtained from RACE PCR was sequenced and deposited to Genbank (DQ386454). Blast search, analysis of the exon-intron positions, analysis of the amino acid sequence, and of conserved domains were performed using NCBI databases. The zbmper signal peptide was predicted with the help of the SignalP 3.0 server (http://www.cbs.dtu.dk). Boxshade (www.ch.embnet.org/software/BOX_form.html) and ClustalW analysis for the generation of a phylogenetic tree (www.es.embnet.org/Doc/phylodendron/clustal-form.html) as well as prediction of glycosylation sites $\left[{ }^{24}\right]$ were performed using commonly accessible software resources.

To confirm the zbmper open reading frame, zbmper cDNA was cloned in frame between the start codon, the subsequent signal peptide and the c-terminal c-Myc6xHis Tag into the pSecTag 2A (Invitrogen) expression vector omitting the zebrafish signal peptide. This expression construct (pSecTagzbmper) was transfected into COS-7 cells using Fugene6 reagent (Roche). After transfection for $48 \mathrm{~h}$ COS-7 cell supernatant was collected and concentrated by 
centrifugation in Centricon spin columns. Proteins were analyzed by western blotting using the anti c-Myc antibody 9E10 (St. Cruz, USA). pSecTagzbmper was also used as a template to generate zbmper RNA with the mMESSAGEmMACHINE T7 Kit from Ambion.

\section{Whole-mount in situ hybridization}

To study the expression pattern of zbmper during embryogenesis, whole mount in situ hybridization was performed as described in the online supplement $\left[{ }^{21}\right]$. Zebrafish embryos were fixed at different developmental stages in 4\% paraformaldehyde and washed in PBST. After dehydration, rehydration and proteinase K (Sigma) treatment embryos were hybridized with antisense zbmper specific probe. After blocking and washing, the embryos were incubated with anti-digoxigenin-AP Fab fragments (Roche). The chromogenic reaction was performed with BM-purple AP substrate (Roche) and levamisol (Sigma). The zbmper dig labeled probe was synthesized using pSPTzbmper that contained a fragment of zbmper between T7 and SP3 priming sites as a template. After appropriate digestion of the plasmid T7 or SP3-RNA polymerase were used to generate the antisense probe or the sense control.

\section{Results}

\section{Cloning and characterization of zbmper mRNA and protein}

To understand the role of BMPER in vertebrate vascular development, we searched for the zebrafish orthologs of mouse BMPER using the NCBI zebrafish genomic database. The complete zbmper cDNA sequence was obtained by 5' and 3' RACE PCR of purified RNA from day-2 wildtype embryos. The obtained zbmper cDNA was sequenced and submitted to the NCBI database (DQ 386454). It consists of 2632 base pairs and contains an ATG start codon partly consistent with Kozak's consensus sequence. A poly A signature (ATTAAA) is present at the 3'end. The open reading frame contains 2007 bases including the TGA stop codon. The zbmper protein consists of 668 amino acids and has a predicted mass of $73.8 \mathrm{kD}$ (Figure 1). Computational analysis identified an $\mathrm{N}$-terminal signal peptide with a cleavage site between amino acids 26 and 27, which indicates extracellular protein secretion. Protein domain analysis revealed that zbmper consists of $5 \mathrm{CR}$ domains $\left[{ }^{25}\right]$, a vWD domain followed by a (TI) domain. This domain organization is identical to that of mBMPER protein. There is a high degree of similarity (75\%) and identity (65\%) between zbmper and mBMPER orthologs. Phylogenetic tree analysis shows that BMPER is highly conserved among vertebrates (Figure 2A). As expected, mammalian BMPER proteins are related more closely to one other than to chicken or fish BMPER. In Drosophila a very similar protein has been reported and named crossveinless2 (dCV2) $\left.{ }^{15}\right]$. Although the TI domain is not present in $\mathrm{dCV} 2$, based on expressional and functional analysis vertebrate BMPER and dCV2 are most likely orthologous species.

\section{zbmper protein expression in vitro}

To determine the size of zbmper we expressed pSecTagzbmper in COS-7 cells (Figure 2B). In the cell supernatant we identified a full length protein of about $85 \mathrm{kD}$ that is somewhat larger than the predicted size. This may be due to glycosylation of zbmper at one or several predicted glycosylation sites located between amino acid 47 through 424 . Additionally, we observed a $42 \mathrm{kD}$ band that denotes a $\mathrm{C}$-terminal cleavage product. We have suggested earlier that mouse BMPER is a cleaved protein $\left[{ }^{12}\right]$ and this appears to be a conserved feature of BMPER orthologs across species.

\section{Spatiotemporal expression of zbmper}

To analyze the spatiotemporal expression of zbmper during zebrafish embryonic development, we performed whole mount in situ hybridization experiments. During early embryogenesis 
(gastrulation) we observed zbmper expression in the ventral half of the embryos (data not shown). At $24 \mathrm{hpf}$, zbmper is expressed along the dorsal midline (dm) of the head (Figure 3A) and in the pineal gland. Strong expression of zbmper was also found in the eye primordia (e) at this developmental stage. Most interestingly, we found intense symmetric zbmper expression in the lateral dorsal aorta associated with the aortic arches, the paraxial mesoderm, and in the otic vesicles. A bridge-like tissue formation expressing zbmper as well as flkl interconnects opposite aortic arch complexes cranially (Figure $3 \mathrm{~A}$ and D). In the trunk region, zbmper is expressed within the intermediate cell mass (ICM) located dorsal to the yolk sac extension and ventral to the notochord on both sides of the embryo (Figure 3B and C). The ICM hosts blood and endothelial cell precursors at this developmental stage [26-28]. The expression pattern of zbmper in the lateral dorsal aorta widely overlaps with $f l k l$ expression at this developmental stage as determined by analysis of $f l k l$ :GFP transgenic zebrafish (Figure 3D). This finding indicates that zbmper is expressed in vascular precursor cells during this stage of development.

At 48 hpf, zbmper continues to be expressed in the dorsal midline of the head caudally to the pineal gland (Figure 3E). Expression in the eye primordia is also still present and at this developmental stage is now predominant at the edge of the lens, an organ which is known for high BMP pathway activity $\left.{ }^{29}\right]$. Most strikingly, zbmper continues to be expressed in the lateral dorsal aorta and the aortic arches. At this more developed stage zbmper expression in the ICM region decreases significantly compared to $24 \mathrm{hpf}$. Well characterized markers of hematopoiesis also show waning expression at this developmental stage $\left[{ }^{26}\right]$.

In sections of zebrafish embryos at $48 \mathrm{hpf}$, we confirmed zbmper expression in the lateral dorsal aorta, aortic arches and lateral plate mesoderm (Figure 3F, G). In more caudal sections zbmper is expressed in the midline in close proximity to the ventral wall of the dorsal aorta. This is the region where hemato- and vasculogenic precursors are located. Several marker proteins for hematopoiesis, including $c-m y b\left[{ }^{30}\right]$ and runxl $\left[{ }^{31,32}\right]$, have been identified in this region between 24 and $48 \mathrm{hpf}$. Expressional and functional properties characterize this region as corresponding to the AGM (aorto-gonad-mesonephros) region in mammals and as the source of adult hematopoiesis in zebrafish $\left[{ }^{33}\right]$.

Taken together, zbmper is expressed in regions known for their high BMP activity such as the pineal gland, the eye lens, and the aortic arches, which fits well to our observation that BMPER interacts with BMP family members $\left[{ }^{12}\right]$. Zbmper is also expressed in areas of known hematoand vasculogenic precursor activity such as the aortic arches, the ICM and the AGM, which is consistent with the differential expression of mBMPER in flk1-positive cells during mouse embryonic development. Thus, expression studies place zbmper at the right place and time to play a role in vasculogenesis and hematopoiesis, as well as to integrate the BMP family into this process during zebrafish development.

\section{Loss of zbmper results in a dorsalized phenotype}

To investigate the function of zbmper during embryogenesis, we injected zbmper specific morpholino oligonucleotides (MOs) at the one or two cell stage into zebrafish embryos. zbmper knockdown was dose dependently efficient to decrease embryonic levels of zbmper protein as determined by western blotting of embryo lysates (Figure 4A) There is a striking relationship between the severity of the phenotype and the amount of residual zbmper protein. As shown in Table I, more than $85 \%$ of zbmper morpholino injected embryos display a dorsalized phenotype at $36 \mathrm{hpf}$ similar to the phenotypes observed in BMP pathway mutants such as swirl (bmp2b) or snailhouse (bmp7) $\left[{ }^{34}\right]$. Both MOs were equally active to generate the dorsalization of the embryos. For MO1 we tested two different dosages and found no significant difference in the total amount of dorsalized embryos. The intensity of dorsalization was shifted towards more severe phenotypes when higher concentrations of zbmper morpholino were used. Injections of unrelated control MO had no effect. The dorsalizing effect 
of zbmper MO injection ranges from weak phenotypes in which tails of injected fish are dysmorphic, shorter in length and display an S-shaped "tail kick" (Figure 4C) to more severe phenotypes (Figure 4D). In all affected embryos the tail fins are hypomorphic and the tips of the tails have lost their original (wildtype) tissue organization. Weakly affected embryos survive at least until 6 days post fertilization (data not shown). Severely dorsalized embryos display pigtail shaped tail deformations with complete loss of tissue organization at the tips of the tails (Figure 4D). Thus, loss of zbmper results in a dorsalized phenotype similar to other BMP agonists. This finding is consistent with a role for zbmper in dorsoventral axis formation as we have reported for mouse BMPER $\left[{ }^{12}\right]$.

\section{Loss of zbmper results in disturbed blood flow and loss of blood cells}

In mildly dorsalized embryos after zbmper knockdown, we noticed that the circulation of blood cells is altered. Most of the blood cells seem to be retained in the trunks of the embryos pulsing slightly back and forth in the rhythm of the beating heart (Figure 5B). More detailed analysis indicated that the blood cell masses are "trapped" within the caudal aorta. Only few cells get into the venous circulation via segmental collaterals whereas the natural outflow via the tail anastomosis between the caudal artery and the caudal vein is obstructed. This finding suggests that blood cells accumulate in the arterial system in weakly dorsalized zbmper morphant embryos as a secondary effect of a disturbed connection between the arterial and venous vascular beds.

In contrast to this relative blood cell accumulation in the trunk, we noticed a very low number of blood cells in more severely dorsalized embryos. Although we could not count absolute numbers of blood cells, we found that the ducts of Cuvier do not carry adequate cell numbers and the sinus venosus - a reservoir of blood cells in wildtype embryos- holds only very few blood cells (Figure 5E). Additionally the caudal blood vessels appear "empty" as compared with wildtype embryos (Figure 5C). To investigate the role of zbmper in blood generation in more detail, we injected gatal:GFP transgenic embryos with zbmper MOs. Uninjected embryos display GFP expression in the ICM at around $24 \mathrm{hpf}$ as shown in Figure 6B. In zbmper MO injected embryos GFP activity was strongly reduced (Figure 6C). This finding is consistent with the low number of circulating blood cells in zbmper morphants. Thus, zbmper is required for normal developmental hematopoiesis to proceed in zebrafish.

\section{Loss of zbmper results in a vascular phenotype}

To study zbmper's effect on blood vessel formation, we injected flkl:GFP embryos with zbmper MOs. These embryos express GFP in a vascular specific manner allowing for direct observation of blood vessel formation using fluorescence microscopy. In our experiments, the generation of endothelial cells was not significantly inhibited in either mild or severe zbmper morphant embryos. While endothelial cells are being generated at apparently normal numbers, the formation of the vascular network is compromised. In the most distal parts of malformed tails the vascular structure is completely abolished (Figure 7C). Instead, a disorganized plexus of endothelial cells is present without vascular lumens or circulating blood cells. Depending on the severity of the phenotype, the caudal vein is altered ranging from dysmorphic vessels (Figure 7B) to complete absence of the caudal vein (Figure 7C, [*]). Most interestingly, the formation and patterning of intersegmental blood vessels (ISV) in the tail is disturbed in loss of zbmper embryos (Figures 7B-E). Instead of running from their ventral origins along the intersomitic boundary to the dorsally located dorsal longitudinal anastomotic vessel (DLAV), a certain number of ISVs penetrate intersomitic boundaries and even cross the adjacent ISV before they connect to the DLAV. In other cases the dorsal parts of ISVs are completely absent and the vessel connects adjacently or turns back ventrally to connect to the main longitudinal vessels. Thus, the ISVs are patent and functional but do not follow the intersomitic boundaries, 
indicating a general disturbance of normal vascular pattering in zbmper knockdown morphant embryos.

\section{Discussion}

We report the cloning of zbmper using mouse BMPER as template for our screening strategy. We demonstrate that zbmper is a conserved protein that is expressed during embryonic development in areas known for high BMP activity and in areas that host hemangiopoietic precursor cells. By loss of function experiments we demonstrate that zbmper is involved in dorso-ventral axis formation and plays a role in the generation of blood cells and in vascular differentiation. These experiments highlight the importance of BMP activity generally, and the tissue-restricted BMP regulator BMPER in particular, in normal hematopoietic and vascular development.

Zbmper is expressed in areas that are known for high BMP activity such as the ventral pole of the embryo, the dorsal midline of the head and the pineal gland $\left[{ }^{35}\right]$, as well as the eye primordia $\left[{ }^{29}\right]$. Most interestingly from the perspective of hematopoietic and vascular development, zbmper is expressed in the aortic arches and in the lateral mesoderm (Figure 3). The aortic arches exhibit active BMP signaling and host vasculogenetic precursor cells $[34,36,37]$. The lateral mesoderm is another important source of vascular precursor cells in embryonic development $\left[{ }^{38}\right]$. Vascular precursor cells migrate from lateral locations within the mesoderm on top of the endodermal layer towards the midline to join the interstitial cell mass (ICM) $\left[{ }^{37}\right]$. The ICM is formed by cells within the mesoderm, originating lateral to the developing somites that express both vascular and blood markers and migrate medially around $18 \mathrm{hpf}$ to fuse at the midline. Cells within the ICM (equivalent to the mammalian yolk sac blood islands) have the potential to differentiate into endothelial cells of the trunk vasculature and into proerythroblasts that begin to enter the circulation around $24 \mathrm{hpf}\left[{ }^{26}\right]$. In whole mount preparations of $24 \mathrm{hpf}$ embryos we detected zbmper expression in the ICM (Figure 3B, C). During embryogenesis, hemangiopoietic capacity of the ICM switches to the AGM region within the first few days of development $\left.{ }^{26}\right]$ as reflected by decreasing expression of gatal in the ICM while expression of $c-m y b$ and runxl increases in the AGM [30,32,39]. The AGM equivalent in zebrafish is found adjacent to the ventral wall of the dorsal aorta. We found zbmper expression in this region at $48 \mathrm{hpf}$. These expression patterns suggest that BMPER may provide a mechanism to coordinate BMP activity during vascular development.

The dorsalized phenotype in zbmper morphants indicates that zbmper acts as a BMP agonist in the zebrafish loss of function model. In our previous gain of function experiments in Xenopus embryos, we observed a strong dorsalizing effect when we injected BMPER RNA in the two ventral blastocysts [ ${ }^{12}$ ]. Injection of BMPER RNA into the two dorsal blastocysts had no effect in those experiments. In zebrafish we also injected zbmper into the yolk sac of embryos but did not observe a clear effect (data not shown). Taking these observations together, both localized overexpression as well as loss of function of BMPER within the whole embryo results in dorsalization. Interestingly, BMPER gain of function activity only has a phenotypic effect when applied at the site of natural BMPER expression (ventrally), which largely overlaps with BMP agonist expression, but not when injected dorsally. This suggests that the antagonistic activity of ectopic BMPER depends on the presence of BMP agonists. In contrast, generalized loss of BMPER protein in zebrafish or Drosophila also antagonizes BMP activity, which suggests that the activity of BMPER may be context dependent. There are at least two explanations for these findings: First, the different BMP-dependent effects of BMPER may in fact reflect differing activities of the cleaved and full length forms, by analogy with twisted gastrulation, another BMP-binding protein, that has different activities in the full length and cleaved forms $\left[{ }^{40}\right]$. Second, BMPER is a protein that is necessary for efficient BMP pathway activation. Loss of function experiments support this notion. On the other hand, excess amounts 
of BMPER may inhibit BMP activity by direct sequestration of BMPs. In this regard it is interesting to note that in regulations of dorso-ventral axis formation, BMPER is dependent on the presence of BMPs but is able to rescue the dorsalizing effect of chordin [ $\left.{ }^{16}\right]$. Future studies will determine which physiologic conditions determine if BMPER acts as an agonist or antagonist of BMP activity.

Because zbmper is expressed in vasculogenic regions in fish embryos, we hypothesized that zbmper would exert a regulatory function in these cells. To study vascular formation in more detail we used transgenic $f l k 1$ :GFP fish lines that allow for direct observation of endothelial cells in the developing embryo. We found that zbmper morphant embryos maintain the capacity to form endothelial cells and to form functional blood vessels. Nonetheless, injected embryos display a vascular phenotype that is most obvious in the tail region. The circulation of blood cells is obstructed in the tails, and blood cells traveling distally become stagnant in the dorsal aorta apparently because the outflow is obstructed. A pulsing mass of blood cells accumulates in the dorsal aorta that in turn appears dilated. In the malformed tail region no normally shaped blood vessels are present in these embryos. Endothelial cells can still be found in the tail tips but they are not arranged properly to form perfused lumenized vasculature. Even in fish with a minimal tail phenotype physiologic circulation is impaired due to abnormal caudal vein formation arguing for a primary vascular defect in zbmper morphants. Although we cannot rule out the possibility that modest changes in endothelial cell numbers are occurring that are below the threshold of our ability to detect, the fact that we observed disturbed blood vessel formation rather than impaired endothelial cell generation indicates that zbmper is involved predominantly in the later phases of vascular remodeling and not significantly in early generation of endothelial cells. The phenotype observed in our studies suggests a qualitative rather than a quantitative effect of BMPER on endothelial cell differentiation.

This notion is also supported by our findings in ISV formation. In normal embryos intersomitic vessels grow closely along the boundaries between somites before interconnecting dorsally $\left[{ }^{20}\right]$. Fate map studies have shown that ISV derive from the lateral plate mesoderm [ $\left.{ }^{38}\right]$. Cells of each ISV leave the aorta only between the ventral regions of adjacent somites, and migrate dorsally to assume one of three ICV cell fates. Most dorsal is a t-shaped cell, based in the dorsal longitudinal anastomotic vessel (DLAV) and branching ventrally; the second constitutes a connecting cell; and the third an inverted T-shaped cell, based in the ventral longitudinal vessel and branching dorsally. The ISV remains between somites during its ventral course, but changes to run mid-somite dorsally, which suggests that the pattern of ISV growth ventrally and dorsally is guided by different cues. In out of bounds $(o b d)$ mutant fish embryos, the ISV formation is disturbed predominantly in the ventral course [ $\left.{ }^{38}\right]$. Loss of zbmper function also causes a mispatterning of the ISVs. They sprout from their regular intersomitic position in the longitudinal vessels but then a number of ISVs sprout without respect to somitic boundaries dorsally and either connect with adjacent ISV from cranial or caudal somites or even cross the next ISV before they connect dorsally to the DLAV. In some cases dorsal parts of ISV are missing whereas the number of origins in the longitudinal vessels is normal. This suggests that in zbmper morphant embryos the ventral signal for ISVs is not affected whereas the dorsal signal is perturbed. How this finding goes along with zbmper's role in BMP signaling or if this function is independent from the BMP pathway remains a topic of further interest. Recent data suggest that BMPER is involved in a vascular phenotype caused by complete inactivation of all FoxO alleles in mouse. In these animals that present massive liver hemangiomas and even angiosarcomas BMPER was found to be highly upregulated in liver endothelial cells underscoring the role of BMPER in blood vessel formation $\left[{ }^{41}\right]$. Interestingly, the Drosophila CV2 phenotype lacks crossveins, which are interconnecting veins between the longitudinal veins of the wing $\left[{ }^{15}\right]$. The phenotype observed in loss of zbmper function zebrafish is remarkably reminiscent of the dCV2 phenotype suggesting a generalized role for BMPER in pattern guidance of interconnecting vessels. 


\section{Supplementary Material}

Refer to Web version on PubMed Central for supplementary material.

\section{Acknowledgements}

We would like to thank Shannon Fishman for excellent technical assistance and fish care. Work in M.M.'s laboratory is supported by the Deutsche Forschungsgemeinschaft (SFB TR 23-A1). Work in J.-W.X.'s laboratory is supported by the NIH (AG 19676). C.P. is supported by grants from the National Institutes of Health (HL61656, HL072347, AG024282, and HL65619) and is an Established Investigator of the American Heart Association and a Burroughs Wellcome Fund Clinical Scientist in Translational Research.

\section{References}

1. Moser M, Patterson C. Bone morphogenetic proteins and vascular differentiation: BMPing up vasculogenesis. Thromb Haemost 2005;94:713-718. [PubMed: 16270622]

2. Ferguson JE 3rd, Kelley RW, Patterson C. Mechanisms of endothelial differentiation in embryonic vasculogenesis. Arterioscler Thromb Vasc Biol 2005;25:2246-2254. [PubMed: 16123328]

3. Jain RK. Molecular regulation of vessel maturation. Nat Med 2003;9:685-693. [PubMed: 12778167]

4. Piccolo S, Sasai Y, Lu B, De Robertis EM. Dorsoventral patterning in Xenopus: inhibition of ventral signals by direct binding of chordin to BMP-4. Cell 1996;86:589-598. [PubMed: 8752213]

5. Valdimarsdottir G, Goumans MJ, Rosendahl A, Brugman M, Itoh S, Lebrin F, et al. Stimulation of Id1 expression by bone morphogenetic protein is sufficient and necessary for bone morphogenetic protein-induced activation of endothelial cells. Circulation 2002;106:2263-2270. [PubMed: 12390958]

6. Langenfeld EM, Langenfeld J. Bone morphogenetic protein-2 stimulates angiogenesis in developing tumors. Mol Cancer Res 2004;2:141-149. [PubMed: 15037653]

7. Winnier G, Blessing M, Labosky PA, Hogan BL. Bone morphogenetic protein-4 is required for mesoderm formation and patterning in the mouse. Genes \& Development 1995;9:2105-2116. [PubMed: 7657163]

8. Morse JH. Bone morphogenetic protein receptor 2 mutations in pulmonary hypertension. Chest 2002;121:50S-53S. [PubMed: 11893684]

9. Urness LD, Sorensen LK, Li DY. Arteriovenous malformations in mice lacking activin receptor-like kinase-1. Nat Genet 2000;26:328-331. [PubMed: 11062473]

10. Li DY, Sorensen LK, Brooke BS, Urness LD, Davis EC, Taylor DG, et al. Defective angiogenesis in mice lacking endoglin. Science 1999;284:1534-1537. [PubMed: 10348742]

11. Yang X, Castilla LH, Xu X, Li C, Gotay J, Weinstein M, et al. Angiogenesis defects and mesenchymal apoptosis in mice lacking SMAD5. Development 1999;126:1571-1580. [PubMed: 10079220]

12. Moser M, Binder O, Wu Y, Aitsebaomo J, Ren R, Bode C, et al. BMPER, a novel endothelial cell precursor-derived protein, antagonizes bone morphogenetic protein signaling and endothelial cell differentiation. Mol Cell Biol 2003;23:5664-5679. [PubMed: 12897139]

13. Coles E, Christiansen J, Economou A, Bronner-Fraser M, Wilkinson DG. A vertebrate crossveinless 2 homologue modulates BMP activity and neural crest cell migration. Development 2004;131:5309_ 5317. [PubMed: 15456729]

14. Binnerts ME, Wen X, Cante-Barrett K, Bright J, Chen HT, Asundi V, et al. Human Crossveinless-2 is a novel inhibitor of bone morphogenetic proteins. Biochem Biophys Res Commun 2004;315:272280. [PubMed: 14766204]

15. Conley CA, Silburn R, Singer MA, Ralston A, Rohwer-Nutter D, Olson DJ, et al. Crossveinless 2 contains cysteine-rich domains and is required for high levels of BMP-like activity during the formation of the cross veins in Drosophila. Development 2000;127:3947-3959. [PubMed: 10952893]

16. Rentzsch F, Zhang J, Kramer C, Sebald W, Hammerschmidt M. Crossveinless 2 is an essential positive feedback regulator of Bmp signaling during zebrafish gastrulation. Development 2006;133:801-811. [PubMed: 16439480]

17. Nasevicius A, Ekker SC. Effective targeted gene 'knockdown' in zebrafish. Nat Genet 2000;26:216220. [PubMed: 11017081] 
18. Westerfield M. The Zebrafish Book: Eugene Press. 1995

19. Kimmel CB, Ballard WW, Kimmel SR, Ullmann B, Schilling TF. Stages of embryonic development of the zebrafish. Dev Dyn 1995;203:253-310. [PubMed: 8589427]

20. Isogai S, Lawson ND, Torrealday S, Horiguchi M, Weinstein BM. Angiogenic network formation in the developing vertebrate trunk. Development 2003;130:5281-5290. [PubMed: 12954720]

21. Mullins MC, Hammerschmidt M, Kane DA, Odenthal J, Brand M, van Eeden FJ, et al. Genes establishing dorsoventral pattern formation in the zebrafish embryo: the ventral specifying genes. Development 1996;123:81-93. [PubMed: 9007231]

22. Crossley PH, Martinez S, Ohkubo Y, Rubenstein JL. Coordinate expression of Fgf8, Otx2, Bmp4, and Shh in the rostral prosencephalon during development of the telencephalic and optic vesicles. Neuroscience 2001;108:183-206. [PubMed: 11734354]

23. Long Q, Meng A, Wang H, Jessen JR, Farrell MJ, Lin S. GATA-1 expression pattern can be recapitulated in living transgenic zebrafish using GFP reporter gene. Development 1997;124:41054111. [PubMed: 9374406]

24. Bairoch A, Bucher P, Hofmann K. The PROSITE database, its status in 1997. Nucleic Acids Res 1997;25:217-221. [PubMed: 9016539]

25. Larrain J, Bachiller D, Lu B, Agius E, Piccolo S, De Robertis EM. BMP-binding modules in chordin: a model for signalling regulation in the extracellular space. Development 2000;127:821-830. [PubMed: 10648240]

26. de Jong JLO, Zon LI. Use of the zebrafish system to study primitive and definitive hematopoiesis. Annual Review of Genetics 2005;39:481-501.

27. Detrich HW 3rd, Kieran MW, Chan FY, Barone LM, Yee K, Rundstadler JA, et al. Intraembryonic hematopoietic cell migration during vertebrate development. Proc Natl Acad Sci U S A 1995;92:10713-10717. [PubMed: 7479870]

28. Kimmel CB, Warga RM, Schilling TF. Origin and organization of the zebrafish fate map. Development 1990;108:581-594. [PubMed: 2387237]

29. Murali D, Yoshikawa S, Corrigan RR, Plas DJ, Crair MC, Oliver G, et al. Distinct developmental programs require different levels of Bmp signaling during mouse retinal development. Development 2005;132:913-923. [PubMed: 15673568]

30. Ballinger CA, Connell P, Wu Y, Hu Z, Thompson LJ, Yin LY, et al. Identification of CHIP, a novel tetratricopeptide repeat-containing protein that interacts with heat shock proteins and negatively regulates chaperone functions. Molecular \& Cellular Biology 1999;19:4535-4545. [PubMed: 10330192]

31. Nishikawa SI, Nishikawa S, Kawamoto H, Yoshida H, Kizumoto M, Kataoka H, et al. In vitro generation of lymphohematopoietic cells from endothelial cells purified from murine embryos. Immunity 1998;8:761-769. [PubMed: 9655490]

32. Kalev-Zylinska ML, Horsfield JA, Flores MVC, Postlethwait JH, Vitas MR, Baas AM, et al. Runx1 is required for zebrafish blood and vessel development and expression of a human RUNX1-CBF2T1 transgene advances a model for studies of leukemogenesis. Development 2002;129:2015-2030. [PubMed: 11934867]

33. Willett CE, Cortes A, Zuasti A, Zapata AG. Early hematopoiesis and developing lymphoid organs in the zebrafish. Dev Dyn 1999;214:323-336. [PubMed: 10213388]

34. Schmid B, Furthauer M, Connors S, Trout J, Thisse B, Thisse C, et al. Equivalent genetic roles for bmp7/snailhouse and bmp2b/swirl in dorsoventral pattern formation. Development 2000;127:957967. [PubMed: 10662635]

35. Knochel S, Dillinger K, Koster M, Knochel W. Structure and expression of Xenopus tropicalis BMP-2 and BMP-4 genes. Mechanisms of Development 2001;109:79-82. [PubMed: 11677055]

36. Nikaido M, Tada M, Saji T, Ueno N. Conservation of BMP signaling in zebrafish mesoderm patterning. Mech Dev 1997;61:75-88. [PubMed: 9076679]

37. Jin SW, Beis D, Mitchell T, Chen JN, Stainier DY. Cellular and molecular analyses of vascular tube and lumen formation in zebrafish. Development 2005;132:5199-5209. [PubMed: 16251212]

38. Childs S, Chen JN, Garrity DM, Fishman MC. Patterning of angiogenesis in the zebrafish embryo. Development 2002;129:973-982. [PubMed: 11861480] 
39. Darrow AL, Fung-Leung WP, Ye RD, Santulli RJ, Cheung WM, Derian CK, et al. Biological consequences of thrombin receptor deficiency in mice. Thrombosis \& Haemostasis 1996;76:860866. [PubMed: 8972001]

40. Larrain J, Oelgeschlager M, Ketpura NI, Reversade B, Zakin L, De Robertis EM. Proteolytic cleavage of Chordin as a switch for the dual activities of Twisted gastrulation in BMP signaling. Development 2001;128:4439-4447. [PubMed: 11714670]

41. Paik JH, Kollipara R, Chu G, Ji H, Xiao Y, Ding Z, et al. FoxOs Are Lineage-Restricted Redundant Tumor Suppressors and Regulate Endothelial Cell Homeostasis. Cell 2007;128:309-323. [PubMed: 17254969]

42. Coffinier C, Ketpura N, Tran U, Geissert D, De Robertis EM. Mouse Crossveinless-2 is the vertebrate homolog of a Drosophila extracellular regulator of BMP signaling. Gene Expr Patterns 2002;2:189194. [PubMed: 12617799] 


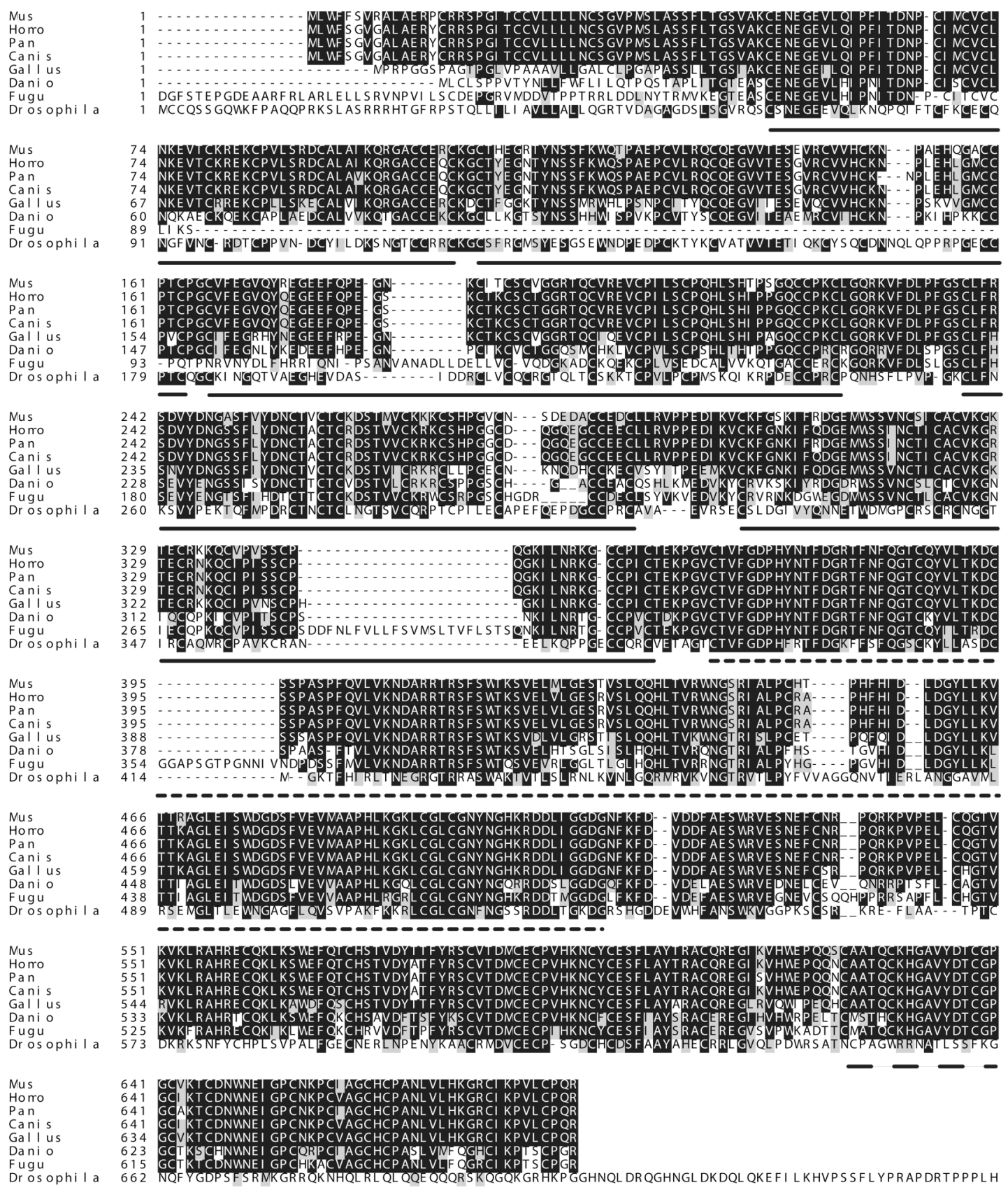

Figure 1. Comparison of orthologous BMPER sequences

Boxshade plot of the amino acid sequences of BMPER orthologs in vertebrates (Mus musculus $\left[{ }^{12,42}\right]$, Homo sapiens [ $\left.{ }^{14}\right]$, Pan troglodytes, Canis familiaris, Gallus gallus [13], Danio rerio, Fugu rubripes, and in Drosophila melanogaster $\left[{ }^{15}\right]$. Black boxes indicate identical amino acids, grey boxes indicate similar amino acids. Solid lines identify vWC domains 1-5, the dotted line denotes the vWD domain and the dashed line indicates the trypsin inhibitor domain (TI). BMPER orthologs share a high degree of homology in vertebrates. The TI domain is absent in Drosophila. 
A

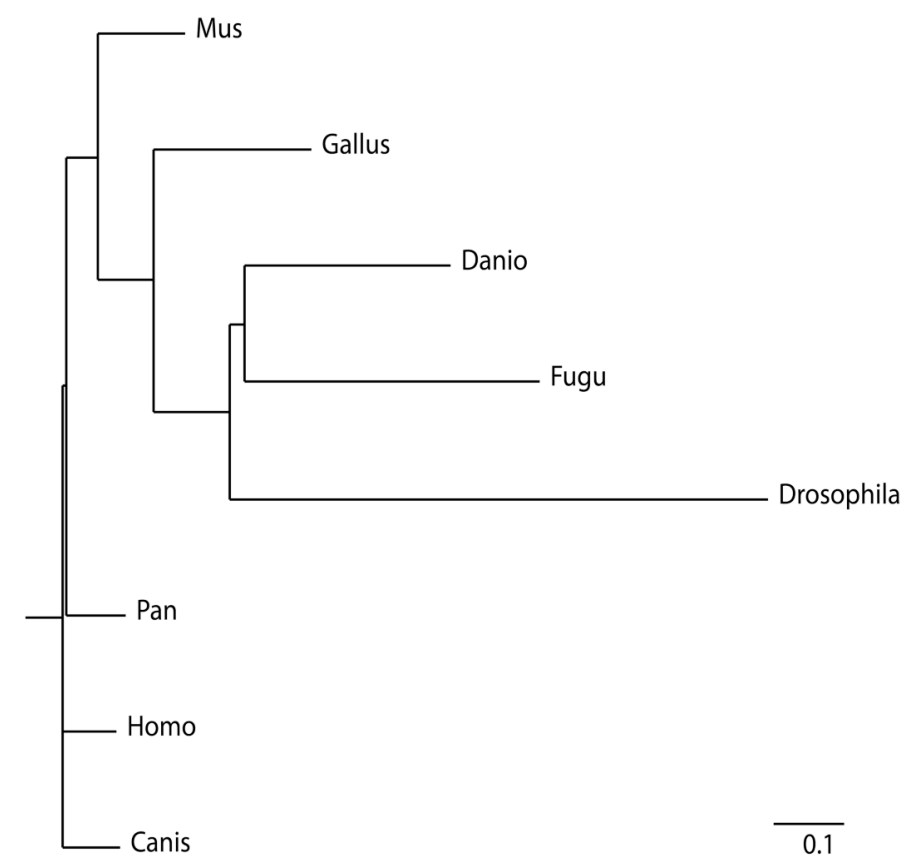

B

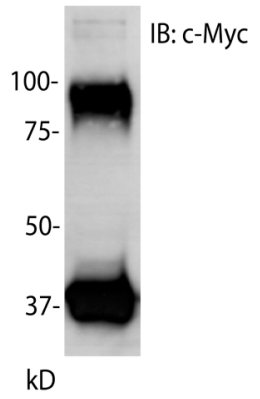

Figure 2. Analysis of BMPER proteins

(A) A phylogenetic tree was calculated for the orthologs shown in Figure 1. The distance of branches from each node indicates increased divergence in sequence similarity among orthologs. The scale represents the fraction of non-identical amino acids along each branch. (B) C-Myc tagged zbmper was analyzed by western blotting on a $12 \%$ SDS-PAGE gel. A full length zbmper protein and a cleaved c-terminal degradation product can be detected. 

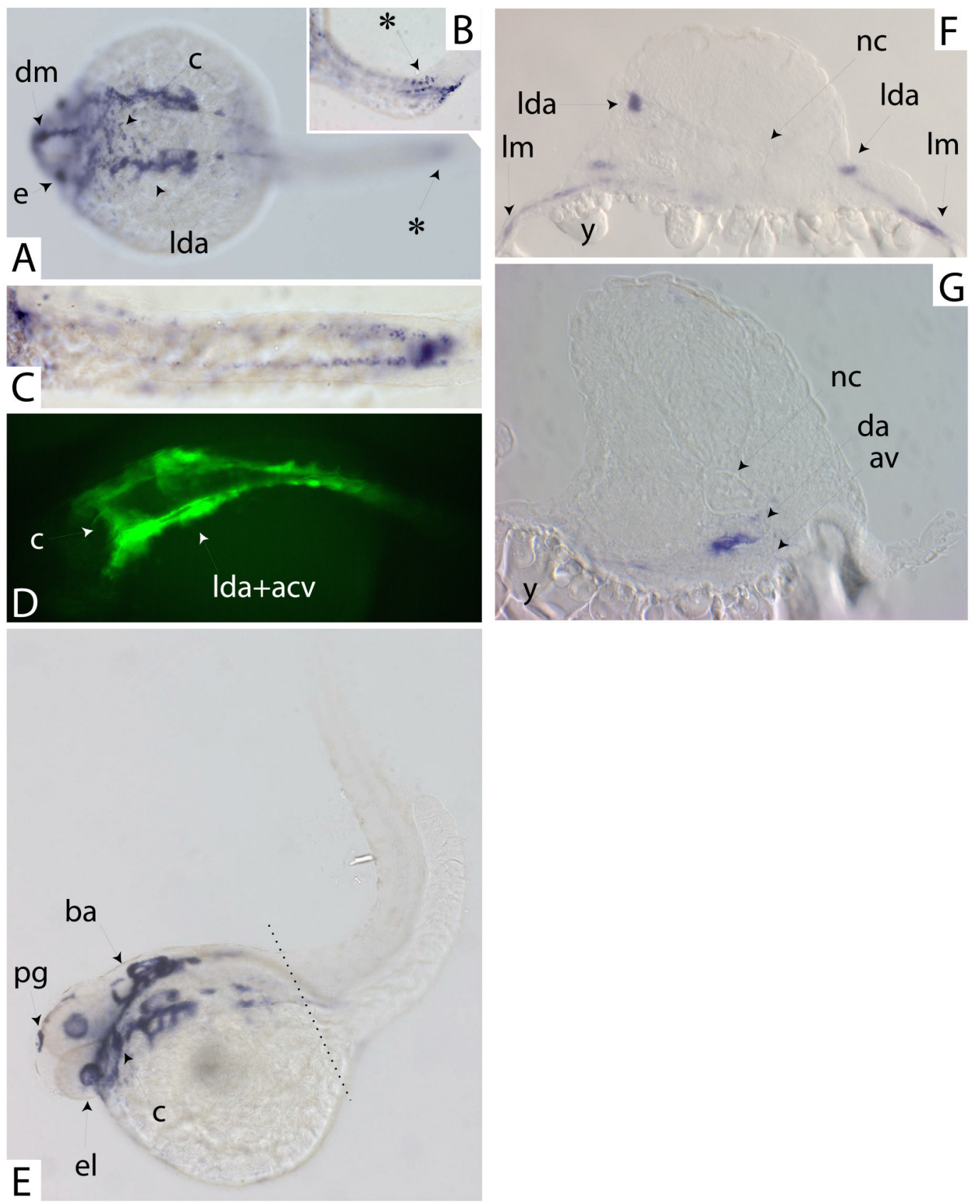

Figure 3. RNA expression of zbmper in whole mount zebrafish embryos

(A-C and E-G) Embryos were analyzed by in situ hybridization using a zbmper specific probe [purple]. (A) Dorsal view of a 24 hpf zebrafish embryo. Zbmper is expressed in the lateral dorsal aorta (lda) associated with the aortic arches as well as within a cranial transverse connection (c), the eye primordia (e), and the dorsal midline (dm) of the head area. (B and C) Higher magnifications of the yolk sac extension. (B) Oblique dorsolateral view, anterior to the left. zbmper is expressed in the ICM (*) located at the distal end of the yolk sac extension. (C) Dorsal view, anterior to the left. Zbmper is expressed in the paraxial ICM more concentrated at the distal end of the yolk sac extension.(D) Dorsolateral view of a $24 \mathrm{hpf} f l k 1$ :GFP transgenic fish embryo shows flk:GFP expression in the symmetric lateral dorsal aorta (lda) and anterior 
cardinal vein (acv) as well as in the transverse connection (c). Anterior to the left. (E) Dorsolateral view of a $48 \mathrm{hpf}$ zebrafish embryo. Zbmper is strongly expressed in the lateral dorsal aorta and the aortic arches. Weaker expression is detectable in the pineal gland (pg) and in the eye lenses (el). (F) Transverse section at the level of the aortic arches at $48 \mathrm{hpf}$. As observed in whole mount preparations zbmper is strongly expressed in the lateral dorsal aorta associated with the aortic arches. Zbmper is also expressed in the lateral mesoderm $(\mathrm{lm})$ that hosts early vascular precursor cells in vertebrate organisms. (G) Transverse section at the level of the dotted line in (E). Zbmper is expressed around the ventral wall of the dorsal aorta but not in the axial vein. (nc) notochord. (da) dorsal aorta. (av) axial vein. (y) yolk sac. 

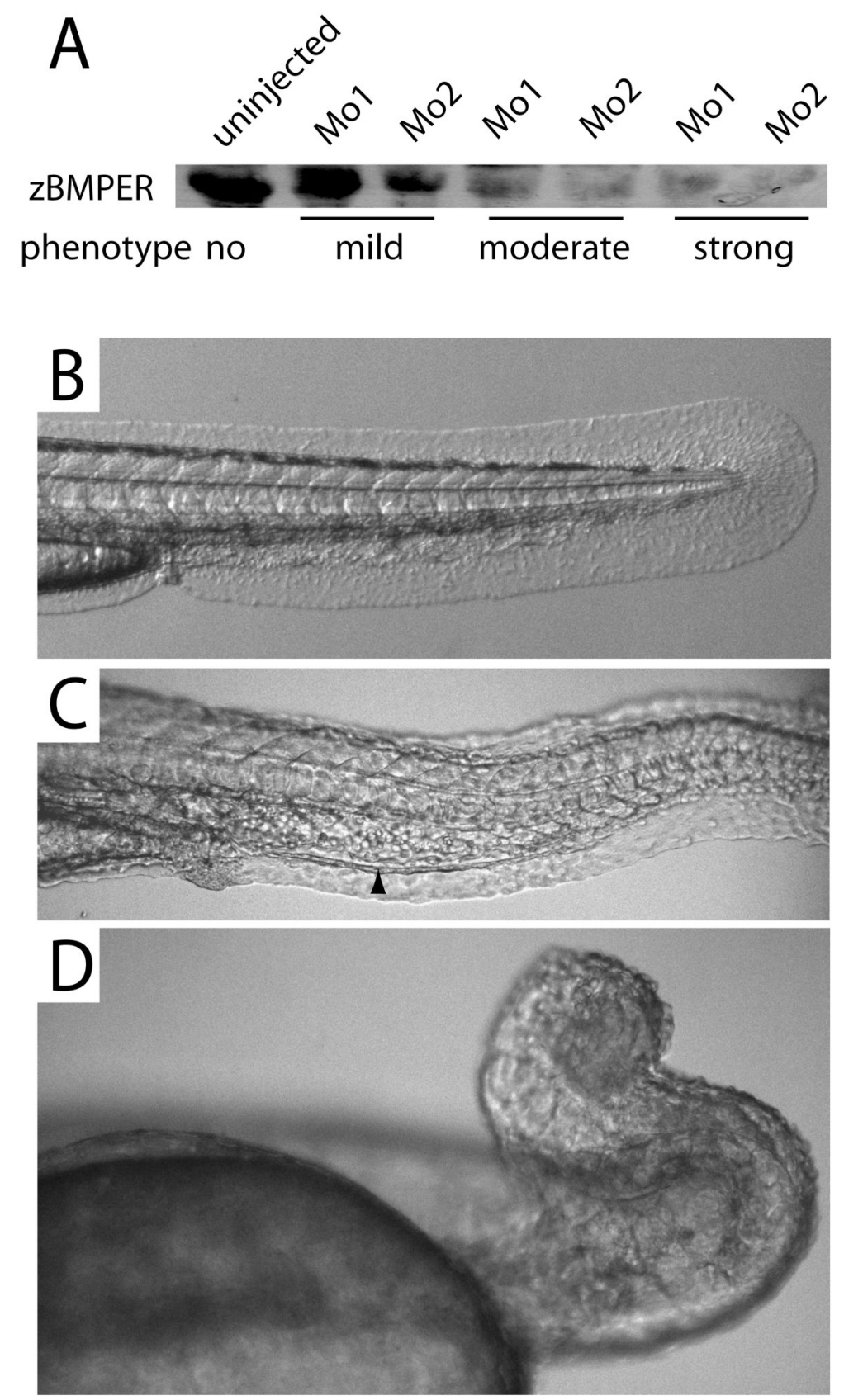

Figure 4. Loss of zbmper causes a dorsalized phenotype

(A) Western blot of zebrafish embryo lysates at 36hpf. Zbmper is effectively knocked down in embryos displaying the characteristic phenotype. (B-D) Lateral views of zebrafish embryos at different developmental stages. (B) Tail of a control zebrafish embryo at $48 \mathrm{hpf}$. (C) Tail of a representative zbmper morphant embryo at $36 \mathrm{hpf}$. The shape of the tail indicates a dorsalized phenotype. Somitic segments distal of the dorsal tail kick are smaller than in controls and are dysmorphic. Blood cells are pooled in the dorsal aorta proximal to the tail deformation (arrowhead). (D) Tail of a zbmper morphant embryo at $72 \mathrm{hpf}$. The tail has adopted a pigtail shape indicating a severely dorsalized phenotype. 

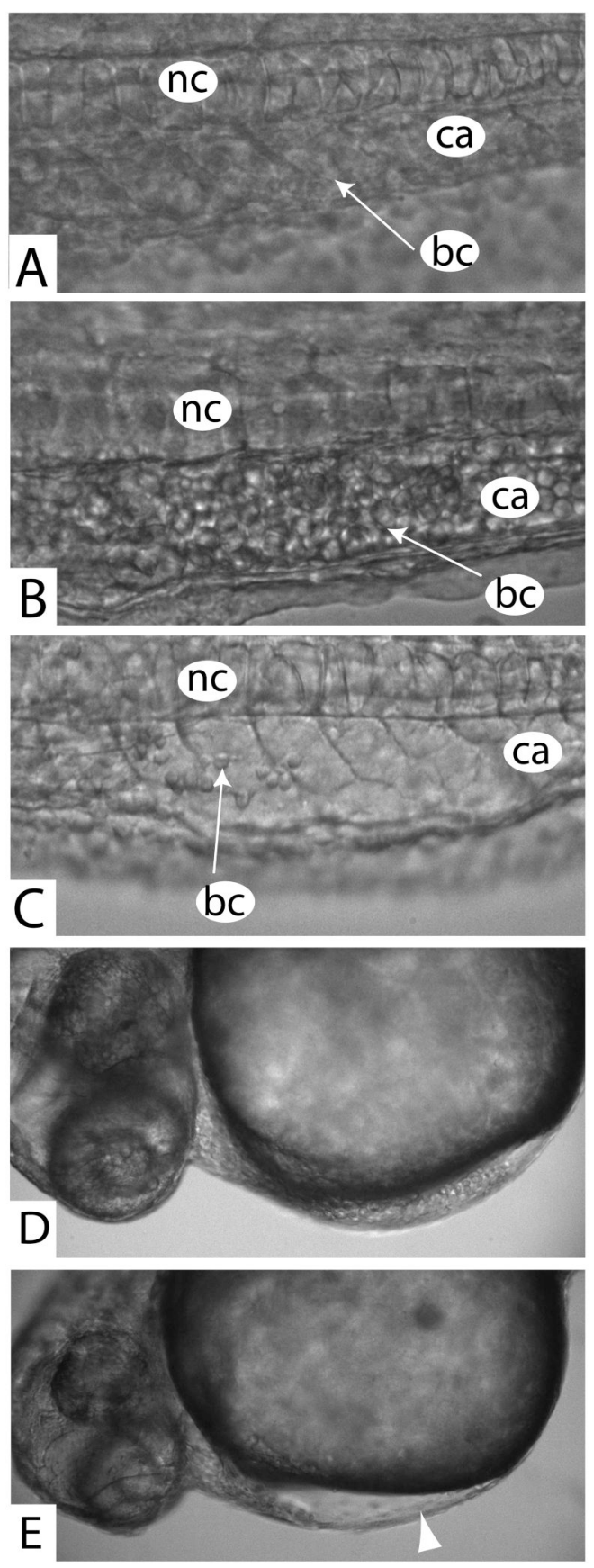

Figure 5. Loss of zbmper results in disturbed blood circulation and loss of blood cells

(A) High magnification of a lateral view of the ventral part of the trunk of a control zebrafish. Anterior to the left. (nc) notochord, (ca) caudal aorta. Note the normal number of circulating blood cells in the dorsal aorta. (B) Lateral view of the trunk of a mildly dorsalized mutant embryo (48 hpf). Note the packed mass of circulating cells within the dorsal aorta. In live fish, this cell mass moves slightly back and forth in the rhythm of the beating heart, suggesting an obstructed outflow from the dorsal aorta. (C) In a more severe phenotype (36 hpf) only a few circulating blood cells (bc) can be observed in the same area of the dorsal aorta. (D) Cranial view of a control embryo at $72 \mathrm{hpf}$. Note the blood cells in the sinus venosus. (E) Cranial view 
of the same embryo as in Figure 4D (72 hpf) displaying a strong loss of blood cells in zbmper morphants. Note only few blood cells in the sinus venosus. 

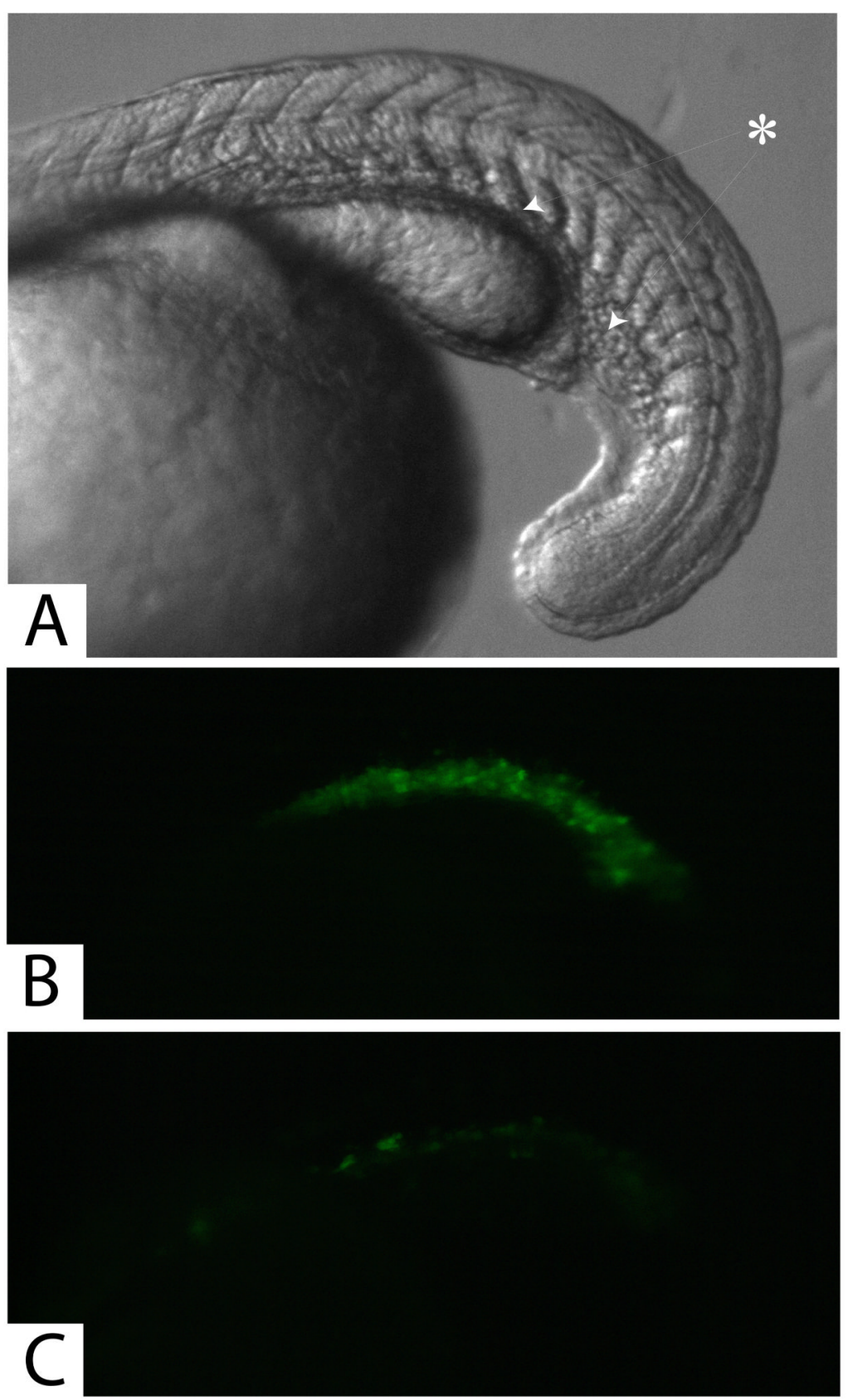

Figure 6. Loss of zbmper reduces gata1 expression in ICM

(A-C) Embryos in identical position. (*) ICM. (A) Tail view (phase) of a control embryo at $20 \mathrm{hpf}$. The ICM extends as indicated by the arrows. (B) Fluorescent image the tail region (as seen in A) of a gatal:GFP transgenic embryo. Note fluorescent signal in the ICM as a reporter for gatal expression. (C) Tail view of a gatal:GFP transgenic zbmper morphant embryo. Note the reduced expression of gatal in the ICM. 

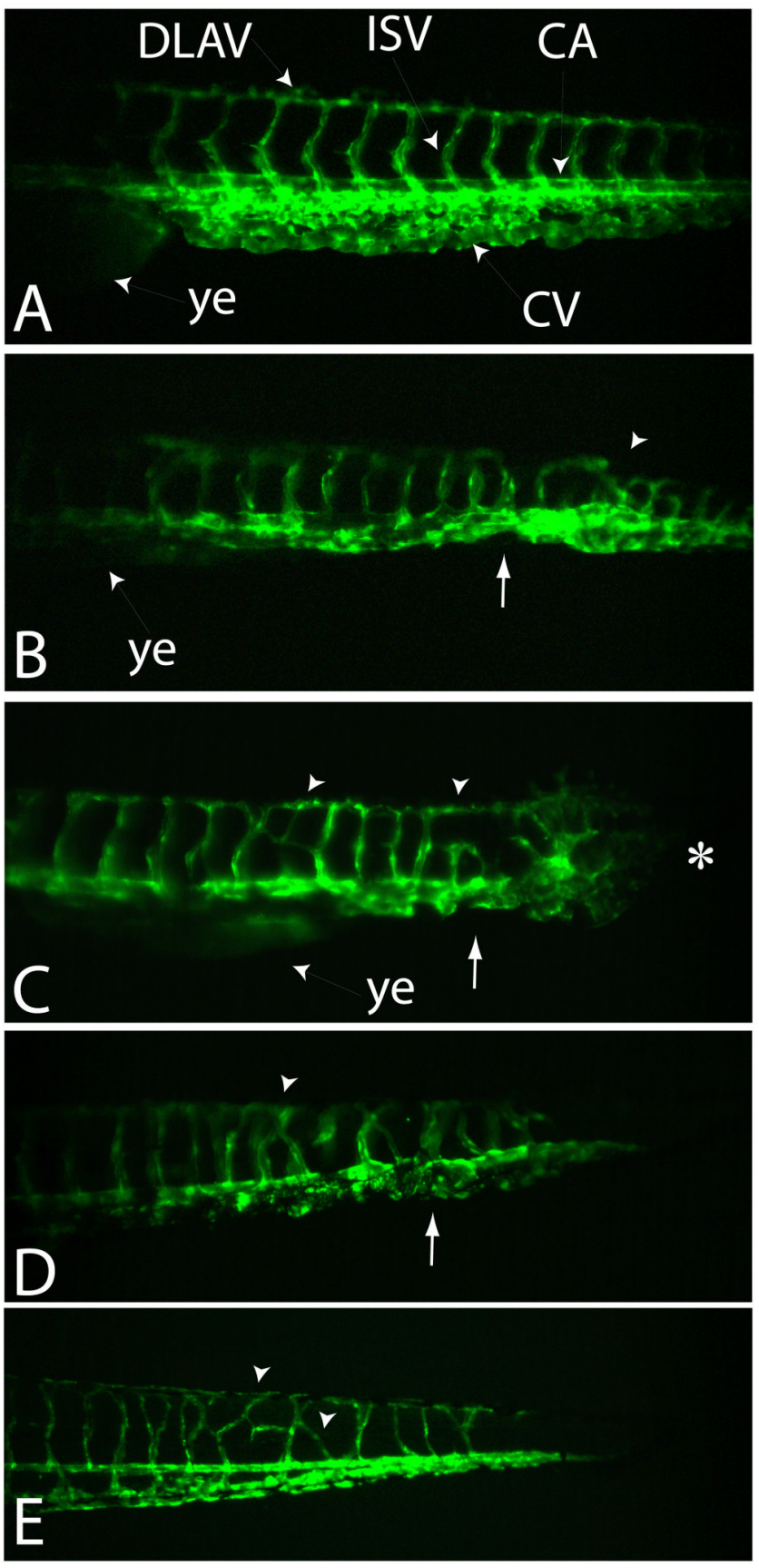

Figure 7. Loss of zbmper results in a vascular phenotype

(A-E) Lateral views of $f l k l$ :GFP transgenic zebrafish embryos at different developmental stages. Anterior to the left. (ye) yolk sac extension. (A) Tail of a control zebrafish embryo at $48 \mathrm{hpf}$. Note the regular, organized patterning of intersegmental blood vessels and the size of the caudal vein plexus. (B-E) zbmper morpholino injected embryos. (B) Tail of zbmper morphant at $48 \mathrm{hpf}$. Note the dysmorphic caudal vein with a localized reduction of vessel size (arrow) and aberrant ISV formation (arrowhead). (C) Tail of a zbmper morphant at $72 \mathrm{hpf}$ displaying a severe tail phenotype. Intersegmental vessels cross segmental boundaries (arrowheads). The caudal vein is absent. At the tip of the tail the normal vascular organization is completely lost (*). In this area endothelial cells are detectable but do not form patent blood 
vessels. (D) Tail of a zbmper morphant at $72 \mathrm{hpf}$ displaying a weak tail phenotype. The indicated intersegmental vessel crosses the respective vessel of the adjacent somite before it connects dorsally. The caudal vein is severely dysmorphic (arrow). (E) Tail of zbmper morphant at $7 \mathrm{dpf}$. Note the absence of the dorsal ends of ISVs and aberrant sprouting. ye = yolk extension, $\mathrm{CA}=$ caudal artery, $\mathrm{CV}=$ caudal vein, $\mathrm{DLAV}=$ dorsal longitudinal anastomotic vessel, ISV = intersegmental vessel. 


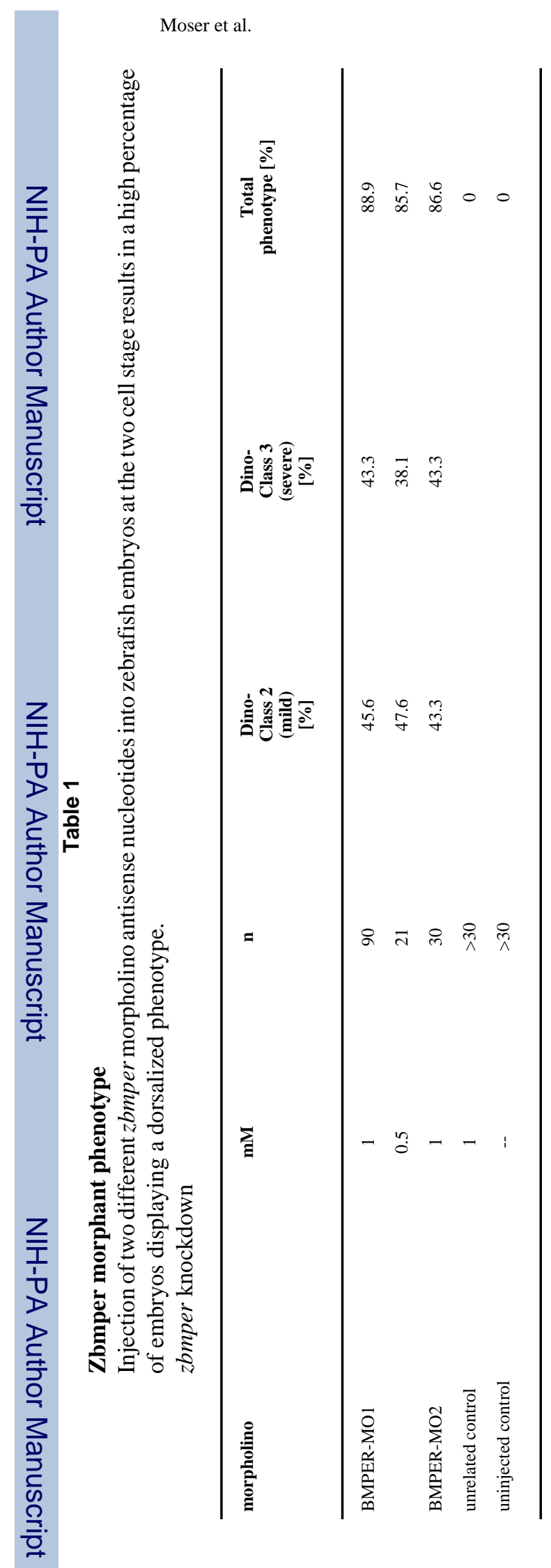

Page 22 